\title{
ENTOMOFAUNA ASSOCIADA AO CULTIVO DE PLEUROTUS SAJOR-CAJU (FR.) SINGER (AGARICALES: AGARICACEAE) NO MUNICÍPIO DO CAPÃO DO LEÃO, RS, BRASIL
}

\author{
E. Bernardii ${ }^{1}$, D.M. Pinto ${ }^{2}$, E.L.G. Costa ${ }^{1}$, J.S. Nascimento ${ }^{1}$
}

${ }^{1}$ Universidade Federal de Pelotas, Instituto de Biologia, Departamento de Microbiologia e Parasitologia, Laboratório de Micologia, CP354, CEP96010-900, Pelotas, RS, Brasil. E-mail: bernardieduardo@yahoo.com.br

\author{
RESUMO
}

\begin{abstract}
O objetivo deste trabalho foi conhecer a diversidade de artrópodes associada ao cultivo de Pleurotus sajor-caju em palha de arroz pasteurizada em ambiente semicontrolado, localizado no sul do Rio Grande do Sul. A captura dos espécimes foi realizada no período de abril a maio de 2007, durante a fase de frutificação da cultura, compreendendo 45 dias. Foram realizadas nove coletas com intervalos de 5 dias, utilizando armadilhas INTRAL AL 012 (12 volts). Capturou-se um total de 3.129 indivíduos divididos em cinco ordens com predominância de Diptera (2.874), Hymenoptera (152), Ortoptera (69), Colembola (59) e Coleoptera (38). A ordem Diptera foi a que apresentou o maior número de famílias, sendo Mycetophilidae (2.530), Sciaridae (264), Cecidomyiidae (10), Phoridae (46), Muscidae (4), Calliphoridae (1) e Culicidae (19).
\end{abstract}

PALAVRAS-CHAVE: Diptera, cogumelos comestíveis, armadilha luminosa, artrópodes.

\section{ABSTRACT}

INSECTS ASSOCIATEDWITHTHE PLEUROTUS SAJOR-CAJU(FR.) SINGER(AGARICALES: AGARICACEAE) CROP IN THE COUNTY OF CAPÃO DO LEÃO, RS, BRAZIL. The objective of this research was to know the diversity of arthropods associated to the Pleurotus sajor-caju crop in rice straw pasteurized in a semicontrolled environment, located in the south of the state of Rio Grande do Sul, Brazil. The capture of specimens was carried out in the period of April through May 2007, during the phase of fruit bodies of the crop, lasting 45 days. Nine collections with intervals of 5 days were carried out, using INTRAL AL 012 (12 volt) traps. A total of 3129 individuals divided into five orders with predominance of Diptera (2874), Hymenoptera (152), Ortoptera (69), Colembola (59) and Coleoptera (38) was captured. The Diptera order was the one that presented the biggest number of families, being Mycetophilidae (2530), Sciaridae (264), Cecidomyiidae (10), Phoridae (46), Muscidae (4), Calliphoridae (1) and Culicidae (19).

KEY WORDS: Diptera, edible mushrooms, luminous trap, arthropods.

\section{INTRODUÇÃO}

Pleurotus spp., pertencente ao filo Basidiomycete, na fase de frutificação, forma estruturas macroscópicas comestíveis. Além dos valores nutricionais, este cogumelo possui atividades antitumoral e imunológica, antimicrobiana, antifúngica, antiviral, entre outras (BRizuela et al., 1998). De acordo com Quimio et al. (1990), o cogumelo P. ostreatus é rico em proteínas, com 27,38\% em base seca. Atualmente, tem-se utilizado a definição destes cogumelos como "cogumelos nutracêuticos" devido ao grande interesse na medicina natural destes como uma alternativa para o tratamento de transtornos fisiológicos e por possuírem diversas características nutricionais (SAVÓN et al., 2002; BONATTI et al., 2004). Além de ser utilizado para alimentação humana, pode ser empregado na alimentação animal, onde Pleurotus spp. coloniza a forragem aumentando seu valor nutritivo (COHEN et al., 2002; SCHIMIDT et al., 2003) e, devido à degradação do substrato este pode ser mais facilmente digerido pelos ruminantes (CASTRO et al., 2004).

Outros cogumelos, como Lentinula edodes, cultivados em toros de Eucalyptus spp. apresentam problemas sanitários como contaminações, tanto no cogumelo como nos toros incubados, geralmente por fungos competidores e causadores de doenças,

${ }^{2}$ Universidade Federal de Pelotas, Instituto de Biologia, Departamento de Microbiologia e Parasitologia, Laboratório de Biologia de Insetos, Pelotas, RS, Brasil. 
seguidos por bactérias, vírus, nematoides e insetos. Entretanto, as maiores perdas na produção de cogumelos, principalmente em regiões tropicais, são atribuídas ao ataque de insetos especialmente em ambientes não totalmente controlados, podendo ocorrer perdas acima de 20\% (BONONI et al., 1995; BUENO et al., 2004).

Figueiredo; Mucci (1985) relataram pragas em Agaricus campestris com destaque para ordem Diptera, famílias Sciaridae, Phoridae, Cecidomyiidae e também, mas com menor expressão, a ordem Lepidoptera e a subclasse Collembola. Já Al-Amidi (1995), em trabalho desenvolvido na República da Irlanda, destacou como principais famílias Sciaridae e Phoridae da ordem Diptera. Assim, o conhecimento sobre os espécimes que ocorrem na cultura de cogumelos permite a identificação prévia de espécies para posterior controle (GASTON et al., 2000; BonHAm et al., 2002; JACTEL et al., 2002).

Este trabalho teve como objetivo conhecer a diversidade de espécimes associada ao cultivo de Pleurotus sajor-caju em palha de arroz pasteurizada em ambiente semicontrolado, localizado no $\mathrm{Mu}$ nicípio de Capão do Leãono sul do RioGrande doSul.

\section{MATERIAIS E MÉTODOS}

Durante este trabalho foram coletados insetos, em armadilha luminosa e armadilha não luminosa, em um módulo de produção de cogumelos de base de produção familiar localizado na Município de Capão do Leão, RS.

O sistema de produção de cogumelos P. sajor-caju, existente na propriedade, obedece uma metodologia em que a palha de arroz seca foi submersa em água por 24 horas, escorrida e pasteurizada em água aquecida a $80-100^{\circ} \mathrm{C}$ durante uma hora. Após o resfriamento, a temperatura ambiente, este substrato foi inoculado manualmente com $3 \%$ de inóculo de $P$. sajor-caju, sendo acondicionado em sacos com volumes de $8 \mathrm{~kg}$ e incubados a temperaturas de $23-28^{\circ} \mathrm{C}$ e umidades relativas de $85-95 \%$ para colonização e frutificação, sendo a colheita realizada de modo manual.

A captura dos espécimes foi realizada no período de abril a maio de 2007, durante a fase de frutificação da cultura, compreendendo 45 dias. Foram realizadas nove coletas com intervalos de 5 dias, sendo expostas duas armadilhas de cada modelo.

A técnica de captura utilizada foi através de armadilha luminosa modelo INTRAL AL 012 (12 volts), provida de lâmpada fluorescente (PINTO et al., 2000; LARANJEIRO, 2003), e de armadilha nãoluminosa, sendo esta diferenciada da primeira pela ausência de lâmpada fluorescente.

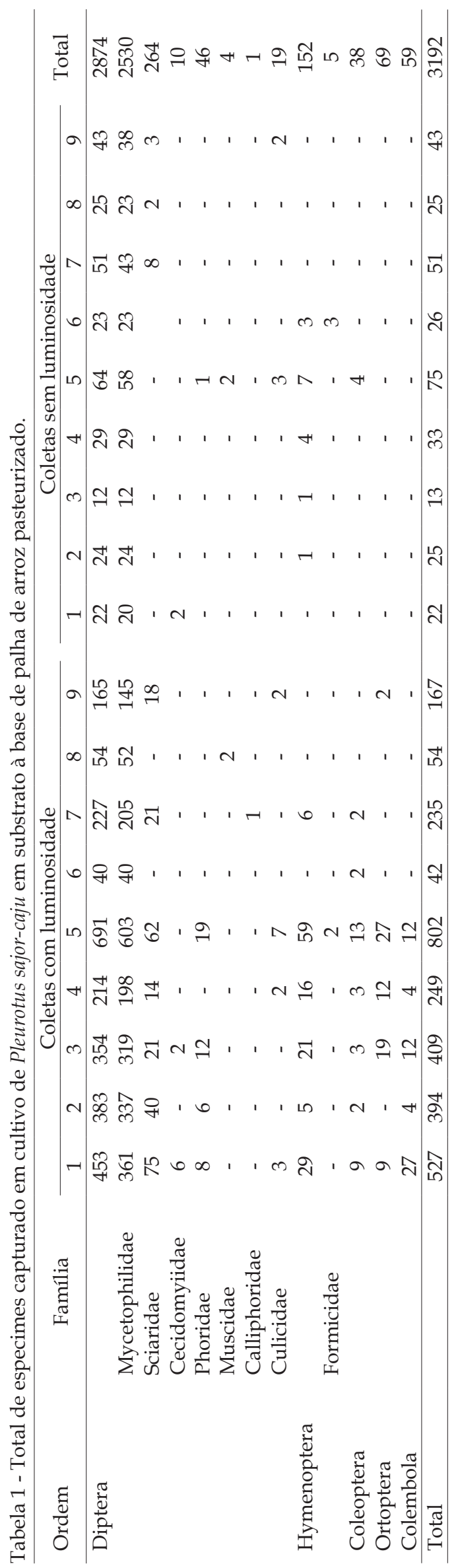


Tabela 2 - Frequência das ordens de Insecta capturadas em armadilhas com e sem luminosidade, durante o ciclo de cultivo de Pleurotus sajor-caju, cultivado em palha de arroz pasteurizada, no Município do Capão do Leão, RS.

\begin{tabular}{|c|c|c|c|c|c|c|}
\hline \multirow{3}{*}{ Ordens } & \multicolumn{6}{|c|}{ Frequência da classe Insecta } \\
\hline & \multicolumn{3}{|c|}{ Com luminosidade } & \multicolumn{3}{|c|}{ Sem luminosidade } \\
\hline & Absoluta (n) & Relativa (\%) & Acumulada (\%) & Absoluta (n) & Relativa (\%) & Acumulada (\%) \\
\hline Diptera & 2.581 & 89,65 & 89,65 & 293 & 93,61 & 93,61 \\
\hline Hymenoptera & 136 & 4,72 & 94,37 & 16 & 5,11 & 98,72 \\
\hline Coleoptera & 34 & 1,18 & 95,55 & 4 & 1,28 & 100,00 \\
\hline Orthoptera & 69 & 2,40 & 97,95 & - & - & - \\
\hline Collembola & 59 & 2,05 & 100,00 & - & - & - \\
\hline Total & 2.879 & 100,00 & & 313 & 100,00 & \\
\hline
\end{tabular}

Tabela 3 - Frequência de famílias da ordem Diptera capturadas em armadilhas com e sem luminosidade, durante o ciclo de cultivo de Pleurotus sajor-caju, cultivado em palha de arroz pasteurizada, no Município do Capão do Leão, RS.

\begin{tabular}{|c|c|c|c|c|c|c|}
\hline \multirow{3}{*}{ Famílias } & \multicolumn{6}{|c|}{ Frequência da classe Insecta } \\
\hline & \multicolumn{3}{|c|}{ Com luminosidade } & \multicolumn{3}{|c|}{ Sem luminosidade } \\
\hline & $\begin{array}{c}\text { Absoluta } \\
\text { (n) }\end{array}$ & Relativa (\%) & Acumulada (\%) & $\begin{array}{l}\text { Absoluta } \\
\text { (n) }\end{array}$ & Relativa (\%) & Acumulada (\%) \\
\hline Mycetophilidae & 2.262 & 87,57 & 87,57 & 270 & 92,15 & 92,15 \\
\hline Sciaridae & 251 & 9,72 & 97,29 & 13 & 4,44 & 96,59 \\
\hline Cecidomyiidae & 8 & 0,31 & 97,60 & 2 & 0,68 & 97,27 \\
\hline Phoridae & 45 & 1,74 & 99,34 & 1 & 0,34 & 97,61 \\
\hline Muscidae & 2 & 0,08 & 99,42 & 2 & 0,68 & 98,29 \\
\hline Calliphoridae & 1 & 0,04 & 99,46 & - & - & - \\
\hline Culicidae & 14 & 0,54 & 100,00 & 5 & 1,71 & 100,00 \\
\hline Total & 2.583 & 100,00 & & 293 & 100,00 & \\
\hline
\end{tabular}

Os espécimes, depois de capturados, foram transferidos para recipientes contendo álcool 70\%, e em seguida transportados até o Laboratório Experimental de Micologia/Departamento de Microbiologia e Parasitologia/Instituto de Biologia/ UniversidadeFederal de Pelotas, onde foram triados e quantificados, e alguns montados e catalogados para acondicionamento no Laboratório de Insetos localizado no referido Departamento.

\section{RESULTADOS E DISCUSSÃO}

Os espécimes coletados estão representados na Tabela 1, onde se verifica um total de3.129 indivíduos divididos em cinco ordens com predominância de Diptera (2.874), Hymenoptera (152), Ortoptera (69), Colembola (59) e Coleoptera (38). A ordem Diptera foi a que apresentou o maior número de famílias, ou seja, sete, a considerar Mycetophilidae (2.530), Sciaridae (264), Cecidomyiidae (10), Phoridae (46), Muscidae (4), Calliphoridae (1) e Culicidae (19). A armadilha luminosa apresentou maiores valores de espécimes capturados ao final da coleta totalizando 2.879 indivíduos, ou seja, valor $89,13 \%$ superior ao coletado com o uso de armadilha nãoluminosa (313).

Ao final das coletas, a classe Insecta foi predominante em ambas as armadilhas, ou seja, com e sem luminosidade. Porém, a frequência relativa da ordem Diptera foi superior na armadilha sem luminosidade $(93,61 \%)$ quando comparada com a luminosa $(89,65 \%)$ mas, em dados absolutos de indivíduos capturados, a armadilha luminosa (2.581) foi superior a não luminosa (293) (Tabela 2). Esses dados podem ser justificados por Costa et al. (2006), os quais citaram o comportamento de fototropismo positivo da maioria dos espécimes da classe Insecta.

As famílias, em número de sete, representantes da ordem Diptera, capturadas durante o período experimental, apresentaram maior número absoluto de espécimes, nas coletas realizadas com a armadilha luminosa, totalizando 2.583 indivíduos, enquanto que na armadilha sem luminosidade foram capturados 293. A família mais representativa foi Mycetophilidae com 2262e270indivíduos capturados nas armadilhas luminosas e sem luminosidade, respectivamente, logo na armadilha sem luminosidade esta família apresentou maior frequência relativa $(92,15 \%)$ frente à luminosa $(87,57 \%)$, fato também observado para as famílias Cecidomyiidae, Muscidae e Culicidae (Tabela 3).

Os resultados deste trabalho são corroborados pelos obtidos por Costa et al. (2006), os quais trabalhando com a entomofauna do cultivo de shiitake (L. edodes), citaram a captura apenas de indivíduos pertencentes à classe Insecta, porém citando dez ordens, onde, Lepidoptera, Homoptera, Thysanoptera, Trichoptera, Hemiptera e Dermaptera foram 
diferentes dos encontrados durante este trabalho. Os mesmos autores citam a família Sciaridae como de maior frequência para o cultivo de shiitake, resultados esses diferentes dos observados neste, onde a família Mycetophilidae foi mais abundante. Estes resultados podem ser justificados pelos diferentes sistemas de cultivo dos cogumelos, onde o shiitake é cultivado em toros de Eucalyptus spp. em ambiente semiprotegido, enquanto que ocultivo de P. sajor-caju foi realizado em substrato natural, pasteurizado e conduzido em ambiente semicontrolado.

Observando a produção de shiitake em toros de eucalipto, no Município deSão Carlos, SP, ZORZENON; PotenZA (2003) constataram queem média $35,17 \%$ dos toros inoculados e em produção, das 15 propriedades amostradas, encontravam-se infestados pela traça Opogona sacchari (Lepidoptera: Tineidae), a qual, segundo os mesmos autores, tem sido constatada inviabilizando cultivos de cogumelos conduzidos por pequenos e médios produtores, pois se trata de um inseto de ampla distribuição e com inúmeros hospedeiros, muito embora não tenha sido identificada durante a realização deste trabalho.

Analisando o cogumelo Amylocystis lapponica, em ambiente de floresta, KomONEN (2001) verificou que este apresentava uma comunidade de 60 espécies de insetos, sendo 37 de Coleoptera, 13 de Diptera, oito de Hymenoptera e duas de Heteroptera, desse modo demonstrando grande diversidade que pode.

Logo, como controle alternativo para insetos de cultivo de cogumelos, especialmente Pleurotus, podem ser utilizadas armadilhas luminosas, bem como a disposição de fitas adesivas, no interior do ambiente de cultivo, apropriadas para a captura de insetos.

\section{CONCLUSÕES}

A maior captura de espécimes ocorre com a utilização de armadilha luminosa.

A ordem Diptera é predominante no cultivo de $P$. sajor-caju em substrato palha de arroz pasteurizado.

A família Mycetophilidae é predominante no cultivo de $P$. sajor-caju, e é capturada em maior número de indivíduos quando utilizadas armadilhas luminosa e não luminosa.

\section{REFERÊNCIAS}

AL-AMIDI, A.H.K. Occurrence of insects and mites in mushroom compost in Ireland. In: ELLIOTT, T.J. (Ed.). Science and cultivation of edible fungi. Roterdam: Balkmam, 1995. p.539-546.
BONATTI, M.; KARNOPP, P.; SOARES, H.M.; FURLAN, S.A. Evaluation of Pleurotus ostreatus and Pleurotus sajor-caju nutritional characteristics when cultivated in different lignocellulosic wastes. Food Chemistry, v.88, p.425-428, 2004.

BONHAM, K.J.; MESIBOV, R.; BASHFORD, R. Diversity and abundance of some ground-dwelling invertebrates in plantation vs. native forests in Tasmania, Australia. Forest Ecology and Management, v.158, n.1/3, p.237-247, 2002.

BONONI, V.L.R.; CAPELARI, M.; MAZIERO, R.; TRUFEM, S.F.B. Cultivo de cogumelos comestíveis. São Paulo: Cone,1995. p.95-104.

BRIZUELA, M.A.; GARCÍA, L.; PÉREZ, L.; MANSUR, M. Basidiomicetos: nueva fonte de metabólitos secundários. Revista Iberoamericana de Micologia, v.15, p.69-74, 1998.

BUENO, F.S.; EIRA, A.F.; FURLAN, M.; NETO, P.F. Natural and organo-synthetic products to control Lentinula edades competitors on Eucalyptus saligna logs. In: ROMAINE, C.P. (Ed.). Edible and medicinal fungi. Miami: USA, 2004. p.431-439.

CASTRO, A.L.A.; PAIVA; P.C.A.; DIAS, E.S.; SANTOS, J. Avaliação das alterações bromatológicas e de degradabilidade do resíduo de lixadeira do algodão após tratamento biológico com Pleurotus sajor-caju. Ciência \& Agrotecnologia, v.28, n.3, p.608-613, 2004.

COHEN, R.; PERSKY, L.; HADAR, Y. Biotechnological applications and potential of wood-degrading mushrooms of the genus Pleurotus. Applied Microbiology and Biotechnology, v.58, n.5, p.582-594, 2002.

COSTA, E.L.G.; BERNARDI, E.; NASCIMENTO, J.S. Entomofauna associada ao cultivo de shiitake [Lentinula edodes (Berk.) Pleger] no município de Arroio do Padre, RS, Brasil. Arquivos do Instituto Biológico, São Paulo, v.73, n.4, p.471-474, 2006.

FIGUEIREDO, M.B.; MUCCI, E.S.F. Doenças e pragas do cogumelo comestível (Agaricus campestris L. EX FR.), In: ENCONTRO NACIONAL SOBRE COGUMELOS COMESTÍVEIS, 1., 1985, Mogi das Cruzes. Anais. Mogi das Cruzes: Instituto de Botânica, 1985. p.69-91.

GASTON, K.J.; BLACKBURN, T.M.; GREENWOOD, J.J.D.; GREGORY, R.D.; QUINN, R.M.; LAWSTON, J.H. Abudance-occupancy relationships. Journal Applied Ecology, v.37: p.39-59, 2000.

JACTEL, H.; GOULART, M.; MENASSIEU, P.; GOUJON, G. Habitat diversity in forest plantations reduces infestations of the pine stem boner Diocyctria sylvestrella. Journal Applied Ecology, v.39, p.618-628, 2002.

KOMONEN, A. Structure of insect communities inhabiting old-growth forest specialist bracket fungi. Ecology Entomology, v.26, n.1, p.63-75, 2001. 
LARANJEIRO, A.J. Estabilidade da entomofauna num mosaico de plantação de eucalipto e áreas naturais de conservação. 2003. 142f. Tese (Doutorado em ciências) - Escola Superior de Agricultura "Luiz de Queiroz", Piracicaba, 2003.

PINTO, R.; JUNIOR, J.S.Z.; FERREIRA, J.A.M.; ZANUNCIO, J.C. Flutuação populacional de coleóptera em plantio de Eucalyptus urophylla no município de Três Marias, Minas Gerais. Floresta E Ambiente, v.7, n.1, p.143-151, 2000.

QUIMIO, T.H.; CHANG, S.T.; ROYSE, D.J. Technical guidelines for mushroom growing in the tropics. Rome: FAO. 1990. 155p.

SAVÓN, R.C.B.; FERNÁNDEZ, C.D.; MANRIQUE, C.E.M.; SEVILLA, E.I.R.; QUEVEDO, H.J.M. Efecto de la luz em la concentración de micoesteroles de Pleurotus ostreatus Var. Florida. Revista Cubana de Alimentación y Nutrición, v.16, n.1, p.13-18, 2002.

SCHMIDT, P.; WECHSLER, F.S.; VARGAS JUNIOR, F.M.; ROSSI, P. Valor nutritivo do feno de braquiária amonizado com uréia ou inoculado com Pleurotus ostreatus. Revista Brasileira de Zootecnia, v.32, n.6, p.18661871, 2003.

ZORZENON, F.J.; POTENZA, M.R. Diagnóstico de danos causados pela traça Opogona sacchari (Lepidoptera: Tineidae) em cultivo de shiitake (Lentinula edodes). Arquivos do Instituto Biológico, São Paulo, v.70, n.4, p.507509, 2003.

Recebido em 13/8/09

Aceito em 16/7/10 